\title{
Comparing Genomes with Duplications: A Computational Complexity Point of View
}

\author{
Guillaume Blin, Cedric Chauve, Guillaume Fertin, Romeo Rizzi, and Stéphane Vialette
}

\begin{abstract}
In this paper, we are interested in the computational complexity of computing (dis)similarity measures between two genomes when they contain duplicated genes or genomic markers, a problem that happens frequently when comparing whole nuclear genomes. Recently, several methods [1], [2] have been proposed that are based on two steps to compute a given (dis)similarity measure $M$ between two genomes $G_{1}$ and $G_{2}$ : First, one establishes a one-to-one correspondence between the genes of $G_{1}$ and the genes of $G_{2}$; second, once this correspondence is established, it explicitly defines a permutation and it is then possible to quantify their similarity using classical measures defined for permutations like the number of breakpoints. Hence, these methods rely on two elements: a way to establish a one-to-one correspondence between genes of a pair of genomes and a (dis)similarity measure for permutations. The problem is then, given a (dis)similarity measure for permutations, compute a correspondence that defines an optimal permutation for this measure. We are interested here in two models to compute a one-to-one correspondence: the exemplar model, where all but one copy is deleted in both genomes for each gene family, and the matching model, which computes a maximal correspondence for each gene family. We show that, for these two models and for three (dis)similarity measures on permutations, namely, the number of common intervals, the maximum adjacency disruption (MAD) number, and the summed adjacency disruption (SAD) number, the problem of computing an optimal correspondence is NP-complete and even APX-hard for the MAD number and the SAD number.
\end{abstract}

Index Terms-Comparative genomics, computational complexity, common intervals, maximum adjacency disruption number, summed adjacency disruption number.

\section{INTRODUCTION}

$\mathrm{T}$ HE comparison of whole genomes from the gene order point of view has been a very active research domain since the early 1990s. In this context, genomes are modeled by sequences of integers, each integer representing a single gene or a genomic marker. ${ }^{1}$ In phylogeny reconstruction, the main problem is thus to compute a (dis)similarity measure between the corresponding integer sequences which approximates the true evolutionary distance between these genomes (see, for instance, [3] for one of the first papers using this approach and [4] for a recent application to vertebrate genomes). Most of the mathematical models developed to compute such (dis)similarity measures are based on the assumption that a given integer appears

1. From now on, we use only the word gene, without loss of generality.

- G. Blin is with the IGM-LabInfo, UMR CNRS 8049, Université de Marnela-Vallée, 77454 Marne-la-Vallée Cedex 2, France.

E-mail: gblin@univ-mlv.fr.

- C. Chauve is with the Department of Mathematics, Simon Fraser University, 8888 University Drive, V5A 1S6, Burnaby (BX), Canada. E-mail: cedric.chauve@sfu.ca.

- G. Fertin is with the Laboratoire d'Informatique de Nantes-Atlantique (LINA), FRE CNRS 2729, Université de Nantes, 2 rue de la Houssiniere, 44322 Nantes Cedex 3, France. E-mail: fertin@lina.univ-nantes.fr.

- R. Rizzi is with the Dipartimento di Matematica e Informatica, Università di Udine, Italy. E-mail: Romeo.Rizzi@simi.uniud.it.

- S. Vialette is with the Laboratoire de Recherche en Informatique (LRI), UMR CNRS 8623, Faculté de Sciences d'Orsay, Université Paris-Sud, 91405 Orsay, France. E-mail: vialette@lri.fr.

Manuscript received 12 July 2006; revised 28 Nov. 2006; accepted 17 Jan. 2007; published online 5 Mar. 2007.

For information on obtaining reprints of this article, please send e-mail to: tcbb@computer.org, and reference IEEECS Log Number TCBBSI-0137-0706. Digital Object Identifier no. 10.1109/TCBB.2007.1069.

$1545-5963 / 07 / \$ 25.00$ C 2007 IEEE exactly once in each considered genome. The rationale of this approach is that genomes are thus simply represented by permutations. However, aside some particular cases such as mitochondrial genomes [3], due to several evolutionary mechanisms (duplication/loss or whole genomes duplications [5]), duplicated genes are very common in genomes. As a result, real data cannot be naturally modeled by permutations.

The first way to overcome such a limitation is to consider genomes at a higher scale than genes, for example, synteny blocks [4]. However, if one wants to stay at the level of genes or, more generally, short genomic markers, one has to deal with the fact that genomes are modeled by sequences of integers where some integers may appear more than once in a given genome. Such genes that appear at several occurrences are said to belong to nontrivial gene families. Two genes represented by the same integer are said to have the same label. Recently, a new two-step permutation-based approach has been proposed for computing (dis)similarity measures between genomes. The first step consists of transforming the two sequences into a single permutation $P$ by establishing a one-to-one correspondence between pairs of genes having the same label (and then, by resorting to renaming procedure, we can always assume that one of the two permutations is the identity permutation, see Section 2). In the second step, a permutation-based (dis)similarity measure is computed from the permutation $P$. The main line of research following this approach seeks the permutation $P$ that optimizes the (dis)similarity measure. The classical criterion retained to define the optimal (dis)similarity measure is the parsimony criterion: One tries to compute the permutation $P$ that induces the maximal (respectively, 
minimal) similarity (respectively, dissimilarity) measure. Note, however, that there exist other methods that are based on the principle of transforming a pair of integer sequences into a permutation but do not aim at optimizing a (dis)similarity measure for the resulting permutation (see [6], [7], [8] for example).

There are two main approaches for computing a one-toone correspondence between two integer sequences. In the exemplar model, introduced by Sankoff [1], for every nontrivial gene family, all but one copy in each genome is deleted. The pair of genes that is conserved for each family is called a pair of ancestral homologs as the goal of the exemplar method is to find the pair of genes which best reflects the original position of the ancestral gene in the common ancestor genome. The matching model is more general as it allows us to conserve more than one copy of a gene family and seeks a maximal one-to-one correspondence between these copies [2]. Several distances have been considered under the exemplar and matching models which are either based on minimizing the number of evolutionary events that allow us to transform a genome into the other, for events like reversals ${ }^{2}$ [1], [9], [10], [11], [12], [13], reversals and insertions and deletions [14], [15], reversals and translocations [16], or on maximizing a similarity measure based on conserved structure in permutations like the number of adjacencies (which is equivalent to minimizing the number of breakpoints) [1], [9], [12], [13], [17] or the number of conserved intervals [18], [19], [20], [21]. As far as we know, none of the above problems has been shown to be solvable in polynomial time and, in fact, most of them have been shown to be NP-complete as soon as duplicates are present in the genomes (see Tables 1 and 2, in Section 6).

In this paper, we present new results on the algorithmic complexity of computing different (dis)similarity measures between pairs of genomes that contain duplicates. We describe results for three (dis)similarity measures, namely, number of common intervals, Maximum Adjacency Disruption (MAD) number, and Summed Adjacency Disruption number (SAD), which will be defined in Section 2. In Section 3, we focus on the problem of computing the number of common intervals in genomes containing duplicates and show that the problem is NP-complete in both the matching and exemplar models. In Sections 4 and 5 , we prove that, under both models, both the MAD and SAD problems are APX-hard when genomes contain duplicates.

\section{Preliminaries}

In this section, we precisely define the three similarity measures we are interested in, together with the exemplar and matching models. As mentioned in the introduction, the three considered measures (number of common intervals, MAD, and SAD) are defined for duplication-free genomes only and, hence, one has to first disambiguate the

2. The reversal model considers signed permutations, where each element has a sign, positive or negative, that indicates which strand on the genome is the corresponding gene located. However, the three (dis)similarity measures we consider in this paper do not take signs into account and, thus, we do not discuss signed permutations here. data by inferring homologs, that is, a nonambiguous mapping between the genes of the two genomes.

We need some notations. A genome is a sequence of unsigned integers. Let $G$ be a genome of size $n$. As mentioned above, a gene family is any integer that occurs in $G$, regardless of its number of occurrences. A gene is an occurrence of a gene family in $G$ and we denote by $G[i]$ the gene that occurs at position $i$ in $G$. Let $\operatorname{occ}(G, g)$ denote the maximum number of occurrences of a gene $g$ in genome $G$ and let $\operatorname{occ}(G)$ be the maximum of $\operatorname{occ}(G, g)$ over all genes $g$ in $G$. The genome $G$ is said to be duplication-free if $\operatorname{occ}(G)=1$. Let $G_{1}$ and $G_{2}$ be two genomes. A matching $\mathcal{M}$ between $G_{1}$ and $G_{2}$ is a set of pairwise disjoint pairs $\mathcal{M}=$ $\left\{\left(i_{1}, j_{1}\right),\left(i_{2}, j_{2}\right), \ldots,\left(i_{k}, j_{k}\right)\right\}$ such that $G_{1}\left[i_{\ell}\right]=G_{2}\left[j_{\ell}\right]$, for all $1 \leq \ell \leq k$. A maximum matching between $G_{1}$ and $G_{2}$ is a matching of maximum cardinality. Suppose that $G$ is duplication-free, let $i$ and $j$ be such that $1 \leq i<j \leq n$ and write $a=G[i]$ and $b=G[j]$. The distance between $a$ and $b$ in $G$, written $\operatorname{Dist}(G, a, b)$, is defined by $\operatorname{Dist}(G, a, b)=|j-i|$.

Given two genomes containing duplications, the first step is thus to establish a nonambiguous mapping between the genes of the two genomes. In the exemplar model, for all gene families, all but one occurrence in each genome is deleted. In other words, we are looking for a matching $\mathcal{M}=$ $\left\{\left(i_{1}, j_{1}\right),\left(i_{2}, j_{2}\right), \ldots,\left(i_{k}, j_{k}\right)\right\}$ between $G_{1}$ and $G_{2}$ such that 1) $G_{1}\left[i_{\ell}\right] \neq G_{1}\left[i_{\ell^{\prime}}\right]$, for all $1 \leq \ell<\ell^{\prime} \leq k$, and 2) each gene family occurs in one pair of $\mathcal{M}$. In the matching model, the goal is to map as many genes as possible, that is, to find a maximum matching between $G_{1}$ and $G_{2}$. The rationale of this preliminary step is that we may now assume that the two genomes are duplication-free. Indeed, suppose the first step results in the matching $\mathcal{M}$, we thus modify the two genomes $G_{1}$ and $G_{2}$ as follows:

1. we delete all genes in $G_{1}$ and $G_{2}$ that are not part of the matching $\mathcal{M}$ and

2. we rename the genes of $G_{1}$ and $G_{2}$ according to the index of the associated pair in $\mathcal{M}$.

Observe that the resulting genomes are both of size $|\mathcal{M}|$. According to the above (for both the exemplar and the matching models) discussion, if a gene family occurs in one genome but not in the other, then all occurrences of this gene family will be deleted in the end. Therefore, we may thus assume in the sequel that any gene family of $G_{1}$ is a gene family of $G_{2}$ and vice versa.

We now turn to precisely defining the three similarity measures in which we are interested. As mentioned before, we assume now that the two genomes are duplication-free, that is, both $G_{1}$ and $G_{2}$ are permutations of size $n$. Moreover, for convenience, by first resorting to an easy renaming procedure, we can always assume that one of the two genomes, say, $G_{1}$, is the identity permutation, that is, $G_{1}=12 \ldots n$.

Number of common intervals. A common interval between $G_{1}$ and $G_{2}$ is a substring of $G_{1}$, that is, a consecutive sequence of genes of $G_{1}$, for which exactly the same content can be found in a substring of $G_{2}$. For example, if $G_{1}=$ 12345 and $G_{2}=14352$, then 1, 2, 3, 4, 5, 3 4, 34 5, 234 5, and 12345 are common intervals. Notice that there exist at least $n+1$ common intervals between $G_{1}$ and $G_{2}$ since each individual gene is always a common interval and $G_{1}$ itself is 
also a common interval. This lower bound is tight, as shown by $G_{1}=1234$ and $G_{2}=2413$. Furthermore, if $G_{1}=G_{2}$, the number of common intervals between $G_{1}$ and $G_{2}$ is $\frac{n(n+1)}{2}$, that is, each possible substring of $G_{1}$ is a common interval.

Maximum Adjacency Disruption (MAD) Number. This notion was introduced by Sankoff and Haque [22]. The MAD number between $G_{1}$ and $G_{2}$, denoted $\operatorname{MAD}\left(G_{1}, G_{2}\right)$, is defined by

$$
\operatorname{MAD}\left(G_{1}, G_{2}\right)=\max \left\{\mathcal{M}_{1}, \mathcal{M}_{2}\right\}
$$

where $\mathcal{M}_{1}=\max \left\{\operatorname{Dist}\left(G_{2}, G_{1}[i], G_{1}[i+1]\right): 1 \leq i \leq n-1\right\}$ and $\mathcal{M}_{2}=\max \left\{\operatorname{Dist}\left(G_{1}, G_{2}[i], G_{2}[i+1]\right): 1 \leq i \leq n-1\right\}$.

The rationale of this double maximization measure lies in the fact that, in general, $\mathcal{M}_{1} \neq \mathcal{M}_{2}$. For instance, if $G_{1}=$ 12345 and $G_{2}=14352$, then $\mathcal{M}_{1}=4$ and $\mathcal{M}_{2}=3$ and, hence, $\operatorname{MAD}\left(G_{1}, G_{2}\right)=\max \{4,3\}=4$.

Summed Adjacency Disruption (SAD) Number. This notion was also introduced by Sankoff and Haque [22] and can be seen as a global variant of the MAD number. The SAD number between $G_{1}$ and $G_{2}$, denoted $\operatorname{SAD}\left(G_{1}, G_{2}\right)$, is defined by

$$
\begin{aligned}
\operatorname{SAD}\left(G_{1}, G_{2}\right)= & \sum_{1 \leq i \leq n-1} \operatorname{Dist}\left(G_{2}, G_{1}[i], G_{1}[i+1]\right) \\
& +\sum_{1 \leq i \leq n-1} \operatorname{Dist}\left(G_{1}, G_{2}[i], G_{2}[i+1]\right) .
\end{aligned}
$$

Going back to our example, $G_{1}=12345$ and $G_{2}=14352$, one obtains

$$
\operatorname{SAD}\left(G_{1}, G_{2}\right)=(4+2+1+2)+(3+1+2+3)=18 .
$$

Of particular importance from a computational complexity point of view, we observe that the MAD and SAD numbers are dissimilarity measures, that is, the associated optimization problem is a minimization one; on the contrary, the number of common intervals is a similarity measure, that is, the associated optimization problem is a maximization one.

\section{Number of Common Intervals}

In this section, we investigate the algorithmic complexity of computing the number of common intervals between two genomes in both the exemplar and matching models. Let ECOMI (respectively, MCOMI) denote the problem of computing the maximum number of common intervals in the exemplar (respectively, matching) model. We show that both ECOMI and MCOMI are NP-complete, even for restricted instances. The proof we give below is valid for both models since it shows NP-completeness in the case where $\operatorname{occ}\left(G_{1}\right)=1$. However, in order to simplify notations, we will mention in the proof only the exemplar model (that is, the ECOMI problem). The proof is made by reduction from VERTEXCOVER. Starting from any instance of VERTEXCOVER (that is, a graph $G=(V, E)$ with $V=\left\{v_{1}, v_{2} \ldots v_{n}\right\}$ and $\left.E=\left\{e_{1}, e_{2} \ldots e_{m}\right\}\right)$, we will first describe a polynomialtime construction of two genomes, $G_{1}$ and $G_{2}$, such that $\operatorname{occ}\left(G_{1}\right)=1$ and $\operatorname{occ}\left(G_{2}\right)=2$. We first describe $G_{1}$ :

$$
\begin{gathered}
G_{1}=b_{1}, b_{2} \ldots b_{m}, x, a_{1}, C_{1}, a_{2}, C_{2} \ldots a_{n}, C_{n}, y, b_{m+n}, \\
b_{m+n-1} \ldots b_{m+1} .
\end{gathered}
$$

The $a_{i} \mathrm{~s}$, the $b_{i} \mathrm{~s}, x$, and $y$ are genes, whereas $C_{i} \mathrm{~s}$ are sequences of genes. They are defined as follows:

- for any $1 \leq i \leq n, a_{i}=2(i-1) m+i$;

- for any $1 \leq i \leq n, C_{i}=\left(a_{i}+1\right),\left(a_{i}+2\right) \ldots\left(a_{i}+2 m\right)$;

- for any $1 \leq i \leq n+m, b_{i}=a_{n}+2 m+i$;

- $x=b_{n+m}+1$; and

- $y=b_{n+m}+2$.

It can be seen that no gene appears more than once in $G_{1}$, thus $\operatorname{occ}\left(G_{1}\right)=1$. Now, we describe the construction of $G_{2}$ :

$$
\begin{gathered}
G_{2}=y, a_{1}, D_{1}^{\prime}, b_{m+1}, a_{2}, D_{2}^{\prime}, b_{m+2} \ldots a_{n-1}, D_{n-1}^{\prime}, b_{m+n-1}, a_{n} \\
D_{n}^{\prime}, b_{m+n}, x
\end{gathered}
$$

The duplicated genes in $G_{2}$ are $b_{1}, b_{2} \ldots b_{n}$ and are spread within the $D_{i}^{\prime}$ s. Moreover, each $b_{i}, 1 \leq i \leq n$, will appear only twice in $G_{2}$. We now describe the contents of $D_{i}^{\prime}$, $1 \leq i \leq n$. Basically, $D_{i}^{\prime}$ is constructed in two steps:

1. We first construct, for each $i$, a sequence of genes $D_{i}$, which is a specific shuffle of the contents of $C_{i}=\left(a_{i}+1\right),\left(a_{i}+2\right) \ldots\left(a_{i}+2 m\right)$. More precisely, let $\min =a_{i}+1$ and $\max =a_{i}+2 m$, then

$$
\begin{aligned}
D_{i}= & \left(a_{i}+3\right),\left(a_{i}+5\right) \ldots\left(a_{i}+2 m-3\right), \\
& \left(a_{i}+2 m-1\right), \min , \max ,\left(a_{i}+2\right),\left(a_{i}+4\right) \ldots \\
& \left(a_{i}+2 m-4\right),\left(a_{i}+2 m-2\right) .
\end{aligned}
$$

2. For any $1 \leq i \leq n$, we obtain $D_{i}^{\prime}$ by adding some $b_{j} \mathrm{~s}$ $(1 \leq j \leq m)$ into $D_{i}$, according to the initial graph $G$ we are given. More precisely, for any edge $e_{j}$ that is incident to a vertex $v_{i}$ in $G$, we add the gene $b_{j}$ between the $j$ th and the $(j+1)$ th gene of $D_{i}$. This process gives us the $D_{i}^{\prime}$ s.

Note that no two $b_{j} \mathrm{~s}(1 \leq j \leq m)$ can appear contiguously in a $D_{i}^{\prime}$ and that no $D_{i}^{\prime}$ starts or ends with a $b_{j}\left(\right.$ all $D_{i}^{\prime} \mathrm{s}$ start and end with a gene that only appears in $C_{i}$ in $G_{1}$ ). In the following, any interval of size one (that is, any singleton), as well as the whole genome, will be called a trivial interval.

Lemma 1. For any exemplar genome $G_{2}^{E}$ of $G_{2}$, the only nontrivial common intervals that occur between $G_{2}^{E}$ and $G_{1}$ are necessarily taken in $G_{1}$ within the sequence $a_{i} C_{i}$, for any $1 \leq i \leq n$.

Proof. We will first prove that, for any exemplar genome $G_{2}^{E}$ obtained from $G_{2}$, any interval of size greater than or equal to 2 that contains $x$ (respectively, $y$ ) also contains $y$ (respectively, $x$ ) and thus corresponds to the whole genome. Suppose, indeed, that there is a common interval, different from a singleton, containing $x$ and not $y$. Let us call this interval $I_{x}$. Now, let us look at what other genes $I_{x}$ could contain in $G_{1}$ :

- If $I_{x}$ contains $b_{m}$ in $G_{1}$, since $b_{m}$ belongs to a $D_{i}^{\prime}$ in $G_{2}^{E}$, this means that $I_{x}$ contains $b_{m+n}$ in $G_{2}^{E}$ and thus contains $y$ in $G_{1}$, a contradiction. 
- If $I_{x}$ contains $a_{1}$ in $G_{1}, I_{x}$ contains, in particular, $b_{m+n}$ in $G_{2}^{E}$ and thus contains $y$ in $G_{1}$, a contradiction.

Hence, any common interval $I_{x}$ that contains $x$ also contains $y$. Now, suppose that a common interval $I_{y}$, different from a singleton, contains $y$ and not $x$ and let us look at what other genes $I_{y}$ could contain in $G_{1}$ :

- If $I_{y}$ contains $b_{m+n}$ in $G_{1}$, then it contains all of the $D_{i}^{\prime} \mathrm{s}$ in $G_{2}^{E}$ and, in particular, it contains all of the $b_{j} \mathrm{~s}, 1 \leq j \leq m$. Thus, it contains $x$ in $G_{1}$, a contradiction.

- If $I_{y}$ contains $a_{n}+2 m$ in $G_{1}$, then it contains, in particular, $b_{m+n-1}$ in $G_{2}^{E}$ and thus contains $b_{m+n}$ in $G_{1}$. We are now back to the previous case.

Hence, the only common interval containing $x$ (respectively, $y$ ) is the whole genome $G_{1}$. Thus, if there are common intervals that are nontrivial, they must be, in $G_{1}$, strictly on the left of $x$, strictly between $x$ and $y$, or strictly on the right of $y$. We will separately investigate these three cases:

1. Intervals strictly on the left of $x$ in $G_{1}$. Since no two $b_{j} \mathrm{~s}, 1 \leq j \leq m$, are contiguous in $G_{2}^{E}$, any such interval would contain at least one gene in a given $D_{i}^{\prime}$, which occurs only in $C_{i}$ in $G_{1}$, a contradiction.

2. Intervals strictly on the right of $y$ in $G_{1}$. Similarly, any such interval would contain an $a_{i}$ in $G_{2}^{E}$, a contradiction.

3. Intervals strictly between $x$ and $y$ in $G_{1}$. Independent of the way $G_{2}^{E}$ is exemplarized, we see that no common interval in $G_{1}$ can contain, at the same time, $a_{i}$ and $a_{i+1}, 1 \leq i \leq n-1$. Thus, the only possible common intervals between $G_{1}$ and $G_{2}^{E}$ must be taken within a given substring of the form $a_{i} C_{i}(1 \leq i \leq n)$ in $G_{1}$ and the lemma is proven.

Lemma 2. For any given $1 \leq i \leq n$, let $\Delta_{i}$ be a subsequence of $D_{i}^{\prime}$ that does not contain any $b_{j}$. If $2 \leq\left|\Delta_{i}\right| \leq 2 m-1$, then it is not a common interval.

Proof. Let $\Delta_{i}$ be a subsequence of $D_{i}^{\prime}$ that does not contain any $b_{j}$ and let $2 \leq\left|\Delta_{i}\right| \leq 2 m-1$. By Lemma $1, \Delta_{i}$ can only be a common interval with a substring of $C_{i}$, which, by construction, contains consecutive integers. Thus, since $\left|\Delta_{i}\right| \geq 2$, it must contain at least two consecutive integers. However, by construction, any two consecutive integers in $D_{i}^{\prime}$ are extremities of an interval that contains both the minimum value $m$ and the maximum value $M$ of $D_{i}^{\prime}$. However, since, in $C_{i}, m$ and $M$ are the left and right extremities, $\Delta_{i}$ is at least as big as $C_{i}$. Since, by construction, $\left|C_{i}\right|=2 \mathrm{~m}$ and since we supposed that $\left|\Delta_{i}\right| \leq 2 m-1$, this cannot happen. Hence, $\Delta_{i}$ is not a common interval.

Lemma 3. For any exemplar genome $G_{2}^{E}$ of $G_{2}$ and for any $1 \leq i \leq n$, only two cases can occur:
1. In $G_{2}^{E}$, all of the $b_{j}$ s have been deleted from $D_{i}^{\prime}$ and, in that case, there are exactly two nontrivial common intervals involving $D_{i}^{\prime}$.

2. In $G_{2}^{E}$, at least one $b_{j}$ has been left within $D_{i}^{\prime}$ and, in that case, there is no nontrivial common interval involving $D_{i}^{\prime}$.

Proof. By Lemma 1, we know that any nontrivial interval is composed in $G_{1}$ of elements of the sequence $a_{i} C_{i}$, for any $1 \leq i \leq n$. Hence, it is composed, in any exemplar genome $G_{2}^{E}$ obtained from $G_{2}$, of elements of the sequence $a_{i} D_{i}^{\prime}$, for any $1 \leq i \leq n$.

Suppose first that all of the $b_{j} \mathrm{~s}$ in $D_{i}^{\prime}$ have been deleted in our exemplar genome $G_{2}^{E}$, thus transforming it into the exemplar subsequence $D_{i}$. By Lemma 1, we know that any nontrivial interval is composed in $G_{1}$ of elements of the sequence $a_{i} C_{i}$, for any $1 \leq i \leq n$. Hence, it is composed, in any exemplar genome $G_{2}^{E}$ obtained from $G_{2}$, of elements of the sequence $a_{i} D_{i}$, for any $1 \leq i \leq n$. In that case, it can be easily seen that, for any $1 \leq i \leq n$ :

1. interval $a_{i} C_{i}$ in $G_{1}$ is a common interval to $a_{i} D_{i}$ in $G_{2}^{E}$ and

2. interval $C_{i}$ in $G_{1}$ is a common interval to $D_{i}$ in $G_{2}^{E}$.

Moreover, by Lemma 2, no strict subsequence $\Delta_{i}$ of $D_{i}$ such that $2 \leq\left|\Delta_{i}\right| \leq\left|D_{i}\right|-1$ is a common interval (we recall that $\left|D_{i}\right|=\left|C_{i}\right|=2 \mathrm{~m}$ by construction). Hence, if all of the $b_{j} \mathrm{~s}$ in $D_{i}^{\prime}$ have been deleted to obtain $D_{i}$, then only two common nontrivial intervals exist in $G_{2}^{E}: a_{i} D_{i}$ (which is common with $a_{i} C_{i}$ in $G_{1}$ ) and $D_{i}$ (which is common with $C_{i}$ in $G_{1}$ ).

Suppose now that at least one $b_{j}$ in $D_{i}^{\prime}$ has not been deleted in $G_{2}^{E}$. First, we note that no nontrivial common interval can include $b_{j}$, since $b_{j}$ does not appear in $C_{i}$. Hence, any possible nontrivial interval involving $D_{i}^{\prime}$ is a substring $\Delta_{i}$ of $D_{i}^{\prime}$ that does not contain any $b_{j}$. However, since no $b_{j}$ is an extremity of $D_{i}^{\prime}$, it implies that, necessarily, $\left|\Delta_{i}\right| \leq 2 m-1$. However, by Lemma 2 , we know that, in that case, $\Delta_{i}$ is not a common interval.

Lemma 4. Let $G$ be a graph and $G_{1}$ and $G_{2}$ be the two genomes obtained by the construction described above. $G$ admits a Vertex Cover $V C$ such that $|V C| \leq k$ iff there exists an exemplar genome $G_{2}^{E}$ obtained from $G_{2}$ having at least $\mathcal{I}=$ $2(n-k)+I_{T}$ common intervals, where $I_{T}$ is the number of trivial common intervals.

Proof. $(\Rightarrow)$ Suppose there exists in $G$ a Vertex Cover $V C$ such that $|V C|=k^{\prime} \leq k$. Let $V C=\left\{v_{i_{1}}, v_{i_{2}} \ldots v_{i_{k^{\prime}}}\right\}$. In $G_{2}$, delete the $b_{j}$ s in the substrings $D_{i}^{\prime}$ for any $i \notin\left\{i_{1}, i_{2} \ldots i_{k^{\prime}}\right\}$. If, after doing this, there remain some $b_{j} \mathrm{~s}$ which appear twice, remove one copy of each arbitrarily. Since, in $\left.G_{2}, 1\right)$ only the $b_{j}$ s are duplicated, 2) each $b_{j}$ occurs exactly twice in $G_{2}$, and 3) $V C$ is a Vertex Cover of $G$, we conclude that, with those deletions, we end up with an exemplar genome $G_{2}^{E}$. In $G_{2}^{E}$, we have at least $(n-k)$ substrings of the form $D_{i}^{\prime}$ for which all of the $b_{j}$ s have been deleted. Thus, by Lemma 3 , we know that they each imply two nontrivial common intervals, which sums up to at least $2(n-k)$. To those intervals, we add the trivial ones. Hence, on the whole, 
we get at least $\mathcal{I}=2(n-k)+I_{T}$ common intervals between $G_{1}$ and $G_{2}^{E}$.

$(\Leftarrow)$ Suppose there exists an exemplar genome $G_{2}^{E}$ obtained from $G_{2}$ and having at least $\mathcal{I}=2(n-k)+I_{T}$ common intervals. Then, there are at least $2(n-k)$ nontrivial common intervals. However, by Lemma 1, we know that they can only occur within the substrings $a_{i} C_{i}, 1 \leq i \leq n$, in $G_{1}$, that is within the substrings $a_{i} D_{i}^{\prime}$, $1 \leq i \leq n$, in $G_{2}^{E}$. By Lemma 3, we know that, in at least $(n-k)$ such substrings, all of the $b_{j} s, 1 \leq j \leq m$, have been deleted. Since $G_{2}^{E}$ is exemplar, this means that the $b_{j}$ s have remained in at most $k$ substrings of the form $a_{i} D_{i}^{\prime}$. By construction, each $b_{j}$ has been included in a $D_{i}^{\prime}$ because the edge $e_{j}$ is incident to the vertex $v_{i}$ in the graph $G$. Since one copy of each $b_{j}$ has remained in $G_{2}^{E}$ and since they are included in at most $k$ substrings of the form $a_{i} D_{i}^{\prime}$, we conclude that those substrings imply a Vertex Cover which is of at most size $k$ in $G$.

As a direct consequence of Lemma 4, we can say that the ECOMI problem is NP-complete. Moreover, as mentioned before, the proof and the result are also valid for the MCOMI problem since our construction implies that $\operatorname{occ}\left(G_{1}\right)=1$. We thus have the following theorem.

Theorem 1. The ECOMI and MCOMI problems are both NPcomplete, even when occ $\left(G_{1}\right)=1$ and occ $\left(G_{2}\right)=2$.

We also consider, for the matching model, instances for which the constraints do not rely on the maximum number of duplicates per family but on the number of families that contain duplicates. With this restriction, we obtain the following result.

Theorem 2. The MCOMI problem is NP-complete even when $f\left(G_{1}\right)=f\left(G_{2}\right)=1$, where $f(G)$ denotes the number of different families of genes that contain duplicates in $G$.

Proof. The proof is directly derived from the proof by Blin and Rizzi [18] in which the authors studied conserved intervals, a measure that is closely related to common intervals. More precisely, a conserved interval is a common interval for which the extremities are conserved [23]. Hence, any conserved interval is by definition a common interval, although the converse is not true in general. However, the construction given in [18] has the property that any common interval is in fact also a conserved interval. Hence, the reduction they provide is also valid for the MCOMI problem, and the result follows.

\section{Maximum Adjacency DisRuption (MAD)}

Let EMAD (respectively, MMAD) denote the problem of computing the minimum MAD number in the exemplar (respectively, matching) model. In this section, we prove inapproximability results for both the EMAD and MMAD problems. More precisely, we show that, for no $\varepsilon>0$, EMAD (respectively, MMAD) admits a $(2-\varepsilon)$-approximation algorithm unless $\mathbf{P}=\mathbf{N P}$. This inapproximability result does not rely on the PCP theorem. We will also remark, however, how reconsidering the reduction proposed in view of the APX-hardness results based on the PCP theorem can one replace the constant 2 above with a strictly bigger constant. The proof is split into two: We first study the complexity of a restricted form of SAT, which we call UNIFORM-SAT, and, in particular, we show that it is NP-complete. Next, we show that a $(2-\varepsilon)$-approximation algorithm for EMAD (respectively, MMAD), for some $\varepsilon>0$, would imply the existence of a polynomial time algorithm for UNIFORMSAT. Finally, we obtain the inapproximability result for EMAD (respectively, MMAD).

In the following, 3SAT will denote the restriction of SAT for which each clause contains at most three literals. We introduce a restricted form of 3SAT called UNIFORM-SAT, as follows: An instance $\langle X, \mathcal{C}\rangle$ of $3 \mathrm{SAT}$ is an instance of UNIFORM-SAT when the following two conditions are met:

1. for each clause $C \in \mathcal{C}$, either all literals occurring in $C$ are positive occurrences of variables from $X$ or all literals occurring in $C$ are negated occurrences of variables from $X$ and

2. for each variable $x \in X, x$ has at most three positive and at most two negated occurrences within $\mathcal{C}$.

A 3SAT formula $F=\bigwedge_{C \in \mathcal{C}} C$ is called 3-bounded if no variable has more than three occurrences within $C$ and is called $(2,2)$-bounded if no variable has more than two positive occurrences and no more than two negated occurrences within $C$. The following two facts are known:

1. the decision problem 3SAT is NP-complete even when restricted to 3-bounded formulas [24] and

2. the optimization problem MAX-3SAT is APX-hard even when restricted to 3-bounded formulas [25].

Since both problems admit a trivial self-reduction in case a variable has only positive (or only negated) occurrences, then the following two facts also hold:

1. 3SAT is NP-complete even when restricted to $(2,2)$-bounded formulas and

2. MAX-3SAT is APX-hard even when restricted to $(2,2)$-bounded formulas.

Notice that, of the above two results, only the second is related to the PCP theorem, whereas the first was known much before its appearance.

The following reduction links the complexity of UNIFORM-SAT to the complexity of $(2,2)$-bounded 3SAT. Given a generic instance $\langle X, \mathcal{C}\rangle$ of $(2,2)$-bounded 3SAT, where $X=\left\{x_{1}, x_{2}, \ldots, x_{n}\right\}$ and $\mathcal{C}=\left\{C_{1}, C_{2}, \ldots, C_{m}\right\}$, consider the instance $\langle Y, \mathcal{P}\rangle$ of UNIFORM-SAT, where $Y=\left\{y_{i}^{j}\right.$ : $i=1,2, \ldots, n, j=0,1,2,3\}$ and $\mathcal{P}=\mathcal{P}_{\text {var }} \cup \mathcal{P}_{\text {cla }}$, where

$$
\begin{gathered}
\mathcal{P}_{\text {var }}=\left\{\left(y_{i}^{j} \vee y_{i}^{j+1 \bmod 4}\right),\left(\neg y_{i}^{j} \vee \neg y_{i}^{j+1 \bmod 4}\right):\right. \\
i=1,2, \ldots, n, j=0,1,2,3\}
\end{gathered}
$$

and $\mathcal{P}_{\text {cla }}=\left\{P_{1}, P_{2}, \ldots, P_{m}\right\}$, where, for $j=1,2, \ldots, m$, the clause $P_{j}$ is obtained from the clause $C_{j}$ as follows: For each literal $\ell$ occurring in $C_{j}$ and assuming that $\ell$ is the $t$ th positive (or the $t$ th negated) literal of variable $x_{i}$ (with $i=1,2, \ldots, n$ and $t=1,2)$ occurring within the clauses $C_{1}, C_{2}, \ldots, C_{m}$ as taken in this order, then the literal $y_{i}^{2 t-1}$ (respectively, the literal $y_{i}^{2 t-2}$ ) is placed in the clause $P_{j}$. In practice, the clause $P_{j}$ is a clause made only of positive literals which is meant to represent the original clause $C_{j}$. 
At the same time, the all-positive or all-negated clauses in $\mathcal{P}_{\text {var }}$ are there to enforce the consistency of the truth values of the variables $y_{i}^{0}, y_{i}^{1}, y_{i}^{2}$, and $y_{i}^{3}$, which are meant to represent either the positive $\left(y_{i}^{1}\right.$ and $\left.y_{i}^{3}\right)$ or the negated $\left(y_{i}^{0}\right.$ and $y_{i}^{2}$ ) occurrences of variable $x_{i}$ within $\mathcal{C}$.

The above is clearly a polynomial time reduction; besides, we have the following lemmas.

Lemma 5. Let $t_{X}: X \mapsto\{0,1\}$ be a truth assignment over $X$ which satisfies at least $c$ of the clauses in $\mathcal{C}$. Then, there exists a truth assignment $t_{Y}: Y \mapsto\{0,1\}$ over $Y$ which satisfies at least $c+8 n$ of the clauses in $\mathcal{P}$.

Proof. Consider the assignment $t_{Y}$ defined by $t_{Y}\left(y_{i}^{1}\right):=$ $t_{Y}\left(y_{i}^{3}\right):=t_{X}\left(x_{i}\right)$ and by $t_{Y}\left(y_{i}^{0}\right):=t_{Y}\left(y_{i}^{2}\right):=\neg t_{X}\left(x_{i}\right)$. Note that each of the $8 n$ clauses in $\mathcal{P}_{\text {var }}$ is satisfied under $t_{Y}$. Moreover, for each $j=1,2, \ldots, m$, the clause $P_{j}$ is satisfied under $t_{Y}$ if and only if the clause $C_{j}$ is satisfied under $t_{X}$.

Lemma 6. Let $t_{Y}: Y \mapsto\{0,1\}$ be a truth assignment over $Y$ which satisfies at least $c+8 n$ of the clauses in $\mathcal{P}$. Then, in polynomial time, we can derive from $t_{Y}$ a truth assignment $t_{X}: X \mapsto\{0,1\}$ over $X$ which satisfies at least $c$ of the clauses in $\mathcal{C}$.

Proof. Truth assignment $t_{Y}$ is called canonical if for each $i=1,2, \ldots, n$, the truth values of the variables $y_{i}^{0}, y_{i}^{1}, y_{i}^{2}$, and $y_{i}^{3}$ are consistent, that is, when $t_{Y}\left(y_{i}^{0}\right)=t_{Y}\left(y_{i}^{2}\right) \neq t_{Y}\left(y_{i}^{1}\right)=t_{Y}\left(y_{i}^{3}\right)$. Notice that by possibly redefining at most two truth values among $t_{Y}\left(y_{i}^{0}\right), t_{Y}\left(y_{i}^{2}\right)$, $t_{Y}\left(y_{i}^{1}\right)$, and $t_{Y}\left(y_{i}^{3}\right)$, we can always assume that $t_{Y}$ is canonical. Indeed, at least two extra clauses from $\mathcal{P}_{\text {var }}$ are satisfied in restoring the consistency among the variables $y_{i}^{0}, y_{i}^{1}, y_{i}^{2}$, and $y_{i}^{3}$, while, at the same time, since at most two truth values have been affected, at most two clauses from $\mathcal{P}_{\text {cla }}$ may lose in satisfaction. In other words, we can make $t_{Y}$ canonical by a majority vote on $y_{i}^{0}, y_{i}^{1}, y_{i}^{2}$, and $y_{i}^{3}$, for each $i=1,2, \ldots, n$, while preserving the fact that at least $c+8 n$ of the clauses in $\mathcal{P}$ are satisfied under $t_{Y}$. Once $t_{Y}$ is canonical, the arguments spent within the proof of the previous lemma are fully reversible.

The above two lemmas imply that UNIFORM-SAT is NP-complete.

Theorem 3. Deciding whether a given UNIFORM-SAT formula is satisfiable is NP-complete.

Theorem 3 here does not need the PCP theorem and is all that is required in the following for proving that, for no $\varepsilon>0$, EMAD (respectively, MMAD) admits a $(2-\varepsilon)$-approximation algorithm unless $\mathbf{P}=\mathrm{NP}$. With dependence on PCP, Lemmas 5 and 6 also imply the following result, which, besides being of independent interest, can be used to show that the right constant for the approximability of EMAD (respectively, MMAD) is not 2.

Theorem 4. Given a UNIFORM-SAT formula, the problem of finding a truth assignment maximizing the number of satisfied clauses is APX-hard.

Proof. We will proceed as follows: Assume we are given a $(1-\varepsilon)$-approximation algorithm $A$ for UNIFORM-SAT and design a $(1-25 \varepsilon)$-approximation algorithm for a $(2,2)$-bounded 3SAT, which rests on algorithm $A$ as a subroutine. The APX-hardness of UNIFORM-SAT then follows from the APX-hardness of a $(2,2)$-bounded 3SAT.

After receiving an instance $\langle X, \mathcal{C}\rangle$ of $(2,2)$-bounded 3SAT in the input, we construct the instance $\langle Y, \mathcal{P}\rangle$ of UNIFORM-SAT, as described above. Assume that the optimal truth assignment $t_{X, o p t}$ for $\langle X, \mathcal{C}\rangle$ satisfies at least opt clauses in $\mathcal{C}$. Clearly, opt $\geq \frac{n}{3}$ since there clearly exists a truth assignment under which, for each variable $x \in X$, at least one of the occurrences of $x$ in $\mathcal{C}$ belongs to a satisfied clause since each clause contains at most three literals. Moreover, by Lemma 5, there exists a truth assignment $t_{Y, o p t}$ over $Y$ satisfying at least $8 n+o p t$ clauses in $\mathcal{P}$. By running algorithm $A$, we are hence guaranteed to find a truth assignment $t_{Y, \text { apx }}$ over $Y$ satisfying at least $(8 n+o p t)(1-\varepsilon)$ clauses in $\mathcal{P}$. Moreover, Lemma 6 (whose proof can be easily converted into a polynomial time algorithm) shows how, starting from this truth assignment $t_{Y, a p x}$, can one obtain a truth assignment $t_{X, \text { apx }}$ over $X$ such that the clauses in $\mathcal{C}$ that are satisfied under $t_{X, a p x}$ are at least

$$
\begin{aligned}
& (8 n+o p t)(1-\varepsilon)-8 n \geq o p t-\varepsilon o p t-8 \varepsilon n \\
& \geq o p t-\varepsilon o p t-24 \varepsilon o p t \geq(1-25 \varepsilon) o p t .
\end{aligned}
$$

We now prove that both the EMAD and MMAD problems are APX-hard. The result holds for both problems since we prove it in the case where $\operatorname{occ}\left(G_{1}\right)=1$, where they coincide. The result rests on a reduction from UNIFORMSAT. Assume we are given an instance $\langle X, \mathcal{C}\rangle$ of UNIFORM-SAT, where $X=\left\{x_{1}, x_{2}, \ldots, x_{n}\right\}$. Here, $\mathcal{C}$ can be partitioned into the family $\mathcal{P}=\left\{P_{1}, P_{2}, \ldots, P_{m_{p}}\right\}$ of clauses comprised of only positive literals and the family $\mathcal{N}=$ $\left\{N_{1}, N_{2}, \ldots, N_{m_{n}}\right\}$ of clauses comprised of only negated literals. Let $M_{\varepsilon}$ be a sufficiently big positive integer that we will later fix in order to force our conclusions. Let us now detail the construction of the two genomes $G_{1}$ and $G_{2}$ from any instance of the UNIFORM-SAT problem. Here, $G_{1}$ is the simple (that is, duplication-free) genome $G_{1}$ of length $L_{1}=2 M_{\varepsilon}+m_{p}+m_{n}+n-1$, defined as follows:

$$
G_{1}=123 \ldots L_{1} \text {. }
$$

A gene at position $i$ in $G_{1}$ with $i \leq m_{p}$ or $i \geq L_{1}-m_{n}+1$ is called an *-gene. Genome $G_{2}$ has length $L_{2}=2 M_{\varepsilon}+6 n-1$ and conforms to the following pattern, where we have found it convenient and pertinent to spot out the displacement of the $*$-genes within genome $G_{2}$ :

$$
\begin{aligned}
G_{2}= & m_{p}+1, m_{p}+2, \ldots, m_{p}+M_{\varepsilon}, *, *, *, *, *, m_{p}+M_{\varepsilon}+1, \\
& *, *, *, *, *, m_{p}+M_{\varepsilon}+2, \ldots \ldots, *, *, *, *, *, \\
& m_{p}+M_{\varepsilon}+n, m_{p}+M_{\varepsilon}+n+1, m_{p}+M_{\varepsilon}+n+2, \\
& \ldots, m_{p}+2 M_{\varepsilon}+n-1 .
\end{aligned}
$$

We will specify later the precise identity of the *-genes within genome $G_{2}$. For now, notice that, in $G_{2}$, we have precisely $n$ runs of five consecutive $*$-genes. We put these runs into a 1,1-correspondence with the $n$ variables in $X$ so that the $i$ th run corresponds to variable $x_{i}$, for $i=1,2, \ldots, n$. For each $i=1,2, \ldots, n$, let $\mathcal{P}_{i}$ and $\mathcal{N}_{i}$ be the lists of index 
sets of the clauses from $\mathcal{P}$ and $\mathcal{N}$ which contain variable $x_{i}$. For example, if $x_{i}$ appears in $P_{3}$, in $P_{7}$, and in $N_{2}$, then $\mathcal{P}_{i}=(3,7)$, whereas $\mathcal{N}_{i}=(2)$. Notice that the lengths of the lists $\mathcal{P}_{i}$ and $\mathcal{N}_{i}$ are at most 3 and 2 , respectively. From the list $\mathcal{P}_{i}$, we obtain a list $\mathcal{P}_{i}^{\prime}$ of length precisely 3 by possibly iterating the last element in $\mathcal{P}_{i}$ the required number of times (that is, $3-\left|\mathcal{P}_{i}\right|$ times). A list $\mathcal{N}_{i}^{\prime}$ of length precisely 2 is similarly obtained from list $\mathcal{N}_{i}$. Now, for each $i=1,2, \ldots, n$, the $i$ th run of five consecutive $*$-genes consists, more precisely, of the following five characters:

$$
\begin{gathered}
(*, *, *, *, *) \rightarrow\left(\mathcal{P}_{i}^{\prime}[1], \mathcal{P}_{i}^{\prime}[2], \mathcal{P}_{i}^{\prime}[3], L_{1}-m_{n}+\mathcal{N}_{i}^{\prime}[1],\right. \\
\left.L_{1}-m_{n}+\mathcal{N}_{i}^{\prime}[2]\right) .
\end{gathered}
$$

The above is clearly a polynomial time reduction. It can also be easily seen that there are no duplications in $G_{1}$, whereas each gene appears at most nine times in $G_{2}$ (that is, $\operatorname{occ}\left(G_{1}\right)=1$ and $\left.\operatorname{occ}\left(G_{2}\right) \leq 9\right)$. Besides, we have the following lemmas.

Lemma 7. Let $t_{X}: X \mapsto\{0,1\}$ be a satisfying truth assignment for $\langle X, \mathcal{C}\rangle$. Then, there exists an exemplar subgenome $G_{2}^{E}$ of $G_{2}$ whose $M A D$ number satisfies $\operatorname{MAD}\left(G_{1}, G_{2}^{E}\right) \leq M_{\varepsilon}+m_{p}+m_{n}+n$.

Proof. For each clause $P_{j} \in \mathcal{P}$, choose a variable $x_{i}$ occurring in $P_{j}$ and such that $t_{X}\left(x_{i}\right)=1$ (remember that $t_{X}$ is a satisfying truth assignment) and color with red one copy of gene $j$ occurring within the $i$ th run of five consecutive *-genes in $G_{2}$. Similarly, for each clause $N_{j} \in \mathcal{N}$, choose a variable $x_{i}$ occurring in $N_{j}$ and such that $t_{X}\left(x_{i}\right)=0$ (again, at least one such variable must exist since $t_{X}$ is a satisfying truth assignment) and color with red one copy of gene $\left(L_{1}-m_{n}\right)+j=2 M_{\varepsilon}+m_{p}+n-1+j$ occurring within the $i$ th run of five consecutive $*$-genes in $G_{2}$. Now, obtain $G_{2}^{E}$ from $G_{2}$ by deleting all of the *-genes except those marked red. Notice that $G_{2}^{E}$ is indeed an exemplar genome on the genes $1,2, \ldots, L_{1}$.

We now verify that

$$
\operatorname{MAD}\left(G_{1}, G_{2}^{E}\right) \leq M_{\varepsilon}+m_{p}+m_{n}+n,
$$

which is better done in two separate steps. First, we check out that any two genes $j$ and $j+1$ that are adjacent in $G_{1}$ are at most $M_{\varepsilon}+m_{p}+m_{n}+n$ positions apart in $G_{2}^{E}$. This follows from the fact that $L_{1}=2 M_{\varepsilon}+m_{p}+$ $m_{n}+n-1$ and considering that the first $M_{\varepsilon}$ positions in $G_{2}^{E}$ are taken by genes $j \in\left[m_{p}+1, m_{p}+M_{\varepsilon}\right]$, whereas the last $M_{\varepsilon}$ positions in $G_{2}^{E}$ are taken by genes $j \in\left[L_{1}-m_{n}-M_{\varepsilon}+1, L_{1}-m_{n}\right]$. Moreover, for

$$
\begin{aligned}
j \in\left[m_{p}+1, m_{p}+M_{\varepsilon}-1\right] \cup & \\
& {\left[L_{1}-m_{n}-M_{\varepsilon}+1, L_{1}-m_{n}-1\right], }
\end{aligned}
$$

genes $j$ and $j+1$ are also adjacent in $G_{2}^{E}$ (more generally, for $j \in\left[m_{p}+1, L_{1}-m_{n}-1\right]$, genes $j$ and $j+1$ both also have a unique occurrence in $G_{2}$, where they are at most six positions apart, and they are at most four positions apart in $G_{2}^{E}$ ). Second and last, we check out that any two genes $i$ and $j$ which are adjacent in $G_{2}^{E}$ are at most $M_{\varepsilon}+$ $m_{p}+m_{n}+n$ positions apart in $G_{1}$. Here, if neither $i$ nor $j$ are $*$-genes, then $i$ and $j$ are also adjacent in $G_{1}$, that is, $j=i \pm 1$. Furthermore, if precisely one among $i$ and $j$, say, $j$, is an *-gene, then $m_{p}+M_{\varepsilon} \leq i \leq m_{p}+M_{\varepsilon}+n$ since, otherwise, $i$ could not be adjacent to an $*$-gene in $G_{2}^{E}$; hence, if $j<i$, then $i-j \leq m_{p}+M_{\varepsilon}+n$, whereas if $i<j$, then $j-i \leq m_{n}+M_{\varepsilon}+n$. Thus, the only interesting case is when both $i$ and $j$ are $*$-genes, that is, both $i$ and $j$ belong either to the interval $\left[1, m_{p}\right]$ or to the interval $\left[L_{1}-m_{n}+1, L_{1}\right]$. It suffices here to notice that, in this case, $i$ and $j$ come from the same interval. Indeed, this follows from the fact that $i$ and $j$ are adjacent in $G_{2}^{E}$ and, hence, correspond to occurrences of the same variable. However, then these two occurrences must either be both positive or both negative since they both have been colored with red in the marking phase.

Lemma 8. For any exemplar genome $G_{2}^{E}$ of $G_{2}$ such that $\operatorname{MAD}\left(G_{1}, G_{2}^{E}\right)<2 M_{\varepsilon}+n$, we can derive in polynomial time from $G_{2}^{E}$ a satisfying truth assignment for $\langle X, \mathcal{C}\rangle$.

Proof. Since $\operatorname{MAD}\left(G_{1}, G_{2}^{E}\right)<2 M_{\varepsilon}+n$, then, in obtaining $G_{2}^{E}$ from $G_{2}$ and for each $i=1,2, \ldots, n$, it must be the case that, in the $i$ th run of five consecutive *-genes in $G_{2}$, either the genes $\mathcal{P}_{i}^{\prime}[1], \mathcal{P}_{i}^{\prime}[2]$, and $\mathcal{P}_{i}^{\prime}[3]$ have all been deleted or the genes $\mathcal{N}_{i}^{\prime}[1]+L_{1}-m_{n}$ and $\mathcal{N}_{i}^{\prime}[2]+L_{1}-$ $m_{n}$ have both been deleted. Consider the truth assignment $t_{X}: X \mapsto\{0,1\}$ such that, for each $i=1,2, \ldots, n$, $t_{X}\left(x_{i}\right)=1$ iff both $\mathcal{N}_{i}^{\prime}[1]+L_{1}-m_{n}$ and $\mathcal{N}_{i}^{\prime}[2]+L_{1}-m_{n}$ have been deleted. We claim that $t_{X}\left(x_{i}\right)$ is a satisfying truth assignment. Indeed, for each clause $P_{j} \in \mathcal{P}$, we know that at least a copy of gene $j$ has been retained in $G_{2}^{E}$. This copy must come from one of the runs of five consecutive $*$-genes in $G_{2}$, say, from the $i$ th run. It follows that $x_{i}$ occurs in $P_{j}$ and that $t_{X}\left(x_{i}\right)=1$. Similarly, for each clause $N_{j} \in \mathcal{N}$, we know that at least a copy of the gene $\left(L_{1}-m_{n}\right)+j$ (that is, of gene $2 M_{\varepsilon}+m_{p}+n-1+j$ ) has been retained in $G_{2}^{E}$. This copy must come from one of the runs of five consecutive *-genes in $G_{2}$, say, from the $i$ th run. It follows that $x_{i}$ occurs in $N_{j}$ and that $t_{X}\left(x_{i}\right)=0$.

Theorem 5. For no $\varepsilon>0$, EMAD (respectively, MMAD) admits $a(2-\varepsilon)$-approximation algorithm unless $\mathbf{P}=\mathbf{N P}$.

Proof. We proceed as follows: We assume that we are given a $(2-\varepsilon)$-approximation algorithm $A$ for EMAD (respectively, MMAD) and design a polynomial time algorithm for UNIFORM-SAT which rests on algorithm $A$ as a subroutine. The theorem then follows from the NP-completeness of UNIFORM-SAT, as stated in Theorem 3. After receiving an instance $\langle X, \mathcal{C}\rangle$ of UNIFORMSAT in the input, we construct the instance $\left\langle G_{1}, G_{2}\right\rangle$ of EMAD (respectively, MMAD), as described above. If $\langle X, \mathcal{C}\rangle$ is satisfiable, then, by Lemma 7 , there exists an exemplar subgenome $G_{2}^{E}$ of $G_{2}$ such that

$$
\operatorname{MAD}\left(G_{1}, G_{2}^{E}\right) \leq M_{\varepsilon}+m_{p}+m_{n}+n .
$$

By running algorithm $A$, we are hence guaranteed to find an exemplar subgenome $G_{a p x}^{E}$ of $G_{2}$ such that

$$
\begin{aligned}
\operatorname{MAD}\left(G_{1}, G_{a p x}^{E}\right) & \leq\left(M_{\varepsilon}+m_{p}+m_{n}+n\right)(2-\varepsilon) \\
& \leq 2 M_{\varepsilon}+2 m_{p}+2 m_{n}+2 n-\varepsilon M_{\varepsilon} .
\end{aligned}
$$


Now, after choosing $M_{\varepsilon} \geq \frac{2 m_{p}+2 m_{n}+2 n}{\varepsilon}$, we conclude that the solution $G_{a p x}^{E}$ produced by algorithm $A$ satisfies $\operatorname{MAD}\left(G_{1}, G_{a p x}^{E}\right) \leq 2 M_{\varepsilon}$. Moreover, Lemma 8 (whose proof can be easily converted into a polynomial time algorithm) shows how, starting from $G_{a p x}^{E}$, one can obtain a satisfying truth assignment for $\left\langle G_{1}, G_{2}\right\rangle$. Conversely, if $\langle X, \mathcal{C}\rangle$ is not satisfiable, then, by Lemma 8 , $\operatorname{MAD}\left(G_{1}, G_{a p x}^{E}\right) \geq 2 M_{\varepsilon}+n$ must hold for the solution returned by algorithm $A$ as it holds for any solution and we can realize that $\langle X, \mathcal{C}\rangle$ was not satisfiable comparing this fact against Lemma 7.

Remark 1. There actually exists a constant $c>2$ such that EMAD (respectively, MMAD) admits no $c$-approximation algorithm unless $\mathbf{P}=\mathbf{N P}$. We can get to this stronger conclusion if, in the proof of Theorem 5 above, we apply Theorem 4 instead of Theorem 3. Moreover, explicit values of $c$ for which this stronger statement holds can also be worked out.

\section{Summed Adjacency DisRuption (SAD)}

Let ESAD (respectively, MSAD) denote the problem of computing the minimum SAD number in the exemplar (respectively, matching) model. In this section, we prove that both problems ESAD and MSAD, expressed on two genomes $G_{1}$ and $G_{2}$ such that $\left|G_{1}\right| \leq\left|G_{2}\right|$, cannot be better than $\log \left(\left|G_{1}\right|\right)$ approximated (here and in the rest of the paper, logarithms are assumed to be base $e$ ). This result holds for both the exemplar and the matching models since we prove it in the case where $\operatorname{occ}\left(G_{1}\right)=1$, for which the two problems coincide. The inapproximability of ESAD (respectively, MSAD) is obtained starting from the inapproximability of SETCOVER. This result will hence depend on the PCP theorem but will deliver stronger SETCOVER-like inapproximability thresholds than for the EMAD and MMAD problems discussed in the previous section.

Let $\langle V, \mathcal{S}\rangle$ be an instance of SETCOVER, where $V=$ $\{1,2, \ldots, n\}$ and $\mathcal{S}=\left\{S_{1}, S_{2}, \ldots, S_{m}\right\}$ is a family of subsets of $V$. We can assume $n$ is even, say, $n=2 k$, and each set $S_{i}$ contains precisely $k=\frac{n}{2}$ elements, say, $s_{1}^{i}, s_{2}^{i}, \ldots, s_{k}^{i}$. The well-known inapproximability results for SETCOVER also hold under these assumptions since we can think of enlarging a groundset $V$, originally on $k$ elements, by adding a set $V^{\prime}$ of $k$ new elements, adding $V^{\prime}$ to $\mathcal{S}$, and enlarging the other sets in $\mathcal{S}$ with elements from $V^{\prime}$ until their size rises up to $k$. Let $M=m^{2} n^{2}$ play the role of a sufficiently big positive integer. Let us now detail the construction of the two genomes, $G_{1}$ and $G_{2}$, from any instance of the SETCOVER problem. Here, $G_{1}$ is a simple (that is, duplication-free) genome of length $L_{1}=M+n+m$ as given by $G_{1}=1,2,3 \ldots L_{1}$. Genome $G_{2}$ has length $L_{2}=$ $M+m(k+1)$ and is constructed as follows:

$$
\begin{aligned}
G_{2}= & n+1, n+2, \ldots, n+M, s_{1}^{1}, s_{2}^{1}, \\
& \ldots, s_{k}^{1}, n+M+1, s_{1}^{2}, s_{2}^{2}, \ldots, s_{k}^{2}, n+M+2, \ldots \\
& \ldots, s_{1}^{m-1}, s_{2}^{m-1}, \ldots, s_{k}^{m-1}, n+M+m-1, s_{1}^{m}, s_{2}^{m}, \ldots, \\
& s_{k}^{m}, n+M+m .
\end{aligned}
$$

The above is clearly a polynomial time reduction; in addition, we have the following lemmas.

Lemma 9. Let $\mathcal{S}^{\prime} \subset \mathcal{S}$ be a set cover of $V$ with $\left|\mathcal{S}^{\prime}\right| \leq s$. Then, there exists an exemplar subgenome $G_{2}^{E}$ of $G_{2}$ whose $S A D$ number satisfies $\operatorname{SAD}\left(G_{1}, G_{2}^{E}\right) \leq 2 s M+5 M$.

Proof. For each element $v \in V$, choose a set $S_{i}$ in $\mathcal{S}^{\prime}$ such that $v \in S_{i}$, that is, $s_{j}^{i}=v$ for some $j=1,2, \ldots, k$. Color with red this copy of gene $v$, that is, the copy of gene $v$ occurring in the position $M+(k+1)(i-1)+j$ of $G_{2}$. Now, obtain $G_{2}^{E}$ from $G_{2}$ by deleting all of the copies of the first $n$ genes, except those marked with red. Notice that $G_{2}^{E}$ is indeed an exemplar genome on the genes $1,2, \ldots, L_{1}$.

We now verify that $\operatorname{SAD}\left(G_{1}, G_{2}^{E}\right) \leq 2 s M+5 M$. Indeed,

$$
\begin{array}{r}
\operatorname{SAD}\left(G_{1}, G_{2}^{E}\right)= \\
\sum_{i=1}^{M+m+n-1} \operatorname{Dist}\left(G_{2}^{E}, G_{1}[i], G_{1}[i+1]\right)+ \\
\sum_{i=1}^{M+m+n-1} \operatorname{Dist}\left(G_{1}, G_{2}^{E}[i], G_{2}^{E}[i+1]\right),
\end{array}
$$

where, assuming that $m$ and $n$ are sufficiently big $(m, n \geq 4)$,

$$
\begin{array}{r}
\sum_{i=1}^{M+m+n-1} \operatorname{Dist}\left(G_{2}^{E}, G_{1}[i], G_{1}[i+1]\right) \leq \\
\sum_{i=1}^{n-1} \operatorname{Dist}\left(G_{2}^{E}, G_{1}[i], G_{1}[i+1]\right)+ \\
(M+m+n)+ \\
\sum_{i=n+1}^{M+n-1} \operatorname{Dist}\left(G_{2}^{E}, G_{1}[i], G_{1}[i+1]\right)+ \\
\leq n(n+m)+(M+m+n)+M+m n \\
\leq 2 M+3 m n^{2} \\
\leq 2 M+m^{2} n^{2} \\
\leq 3 M,
\end{array}
$$

and where, again assuming that $m$ and $n$ are sufficiently big $(m, n \geq 4)$, 


$$
\begin{array}{r}
\sum_{i=1}^{M+m+n-1} \operatorname{Dist}\left(G_{1}, G_{2}^{E}[i], G_{2}^{E}[i+1]\right)= \\
\sum_{i=1}^{M-1} \operatorname{Dist}\left(G_{1}, G_{2}^{E}[i], G_{2}^{E}[i+1]\right)+ \\
\sum_{i=M}^{M+m+n-1} \operatorname{Dist}\left(G_{1}, G_{2}^{E}[i], G_{2}^{E}[i+1]\right)= \\
\quad(M-1)+ \\
\leq M+\sum_{S_{i} \notin \mathcal{S}^{\prime}}^{M+m+n-1} \operatorname{Dist}\left(G_{1}, G_{2}^{E}[i], G_{2}^{E}[i+1]\right) \\
\leq M+m+2 s\left(M+m+n+n^{2}\right) \\
\leq M+2 s M+m^{2} n^{2} \\
\leq 2 s M+2 M .
\end{array}
$$

To better explain the upper bound on the term $\sum_{i=M}^{M+m+n-1} \operatorname{Dist}\left(G_{1}, G_{2}^{E}[i], G_{2}^{E}[i+1]\right)$ used in the above chain of inequalities, denote with $p_{i}, i=0,1, \ldots, m$, the absolute position of the gene $M+n+i$ inside the genome $G_{2}^{E}$. (Thus, $p_{0}=M$ and $p_{n}=M+n+m$ ). Clearly,

$$
\begin{gathered}
\sum_{i=M}^{M+m+n-1} \operatorname{Dist}\left(G_{1}, G_{2}^{E}[i], G_{2}^{E}[i+1]\right)= \\
\sum_{i=1}^{m} \sum_{j=p_{i-1}}^{p_{i}-1} \operatorname{Dist}\left(G_{1}, G_{2}^{E}[j], G_{2}^{E}[j+1]\right) .
\end{gathered}
$$

Now, when $S_{i} \notin \mathcal{S}^{\prime}$, then the two genes $n+M+(i-1)$ and $n+M+i$ are adjacent both in $G_{2}^{E}$ and in $G_{1}$, where $\sum_{j=p_{i-1}}^{p_{i}-1} \operatorname{Dist}\left(G_{1}, G_{2}^{E}[j], G_{2}^{E}[j+1]\right)=1$. Also, for each $i=1,2, \ldots, m$,

$$
\operatorname{Dist}\left(G_{1}, G_{2}^{E}\left[p_{i-1}\right], G_{2}^{E}\left[p_{i-1}+1\right]\right) \leq M+m+n
$$

and $\operatorname{Dist}\left(G_{1}, G_{2}^{E}\left[p_{i}-1\right], G_{2}^{E}\left[p_{i}\right]\right) \leq M+m+n$ since $M+m+n$ is the length of $G_{1}$. Furthermore, $p_{i}-p_{i-1} \leq 1+k \leq n$, and, for each

$$
\begin{aligned}
& j=1,2, \ldots, p_{i}-p_{i-1}-2, \\
& \operatorname{Dist}\left(G_{1}, G_{2}^{E}\left[p_{i-1}+j\right], G_{2}^{E}\left[p_{i-1}+j+1\right]\right) \leq n .
\end{aligned}
$$

Lemma 10. For any exemplar subgenome $G_{2}^{E}$ of $G_{2}$ such that $\operatorname{SAD}\left(G_{1}, G_{2}^{E}\right)<2 s M$, we can derive, from $G_{2}^{E}$ and in polynomial time, a set cover $\mathcal{S}^{\prime} \subset \mathcal{S}$ of $V$ such that $\left|\mathcal{S}^{\prime}\right| \leq s$.

Proof. Let $\mathcal{S}^{\prime}$ be the family of those $S_{i} \in \mathcal{S}$ for which there exists a $v \in S_{i}$, say, $v=s_{i}^{j}$, such that, in obtaining $G_{2}^{E}$ from $G_{2}$, the copy $s_{i}^{j}$ of gene $v$ has not been deleted. Notice that $\mathcal{S}^{\prime}$ is a cover of $V$ since all genes $1,2, \ldots, L_{1}$ occur in $G_{2}^{E}$. Moreover, $\left|\mathcal{S}^{\prime}\right| \leq s$ follows from $\operatorname{SAD}\left(G_{1}, G_{2}^{E}\right)<2 s M$. Indeed,

$$
\sum_{i=M}^{M+m+n-1} \operatorname{Dist}\left(G_{1}, G_{2}^{E}[i], G_{2}^{E}[i+1]\right) \leq S A D\left(G_{1}, G_{2}^{E}\right) \leq 2 s M .
$$

However, for every $i$ such that $S_{i} \in \mathcal{S}^{\prime}$, the genes $M+$ $n+(i-1)$ and $M+n+i$ are not consecutive in $G_{2}^{E}$. Let us denote with $p_{i-1}$ and $p_{i}$ the absolute positions of genes $M+n+(i-1)$ and $M+n+i$ within the genome $G_{2}^{E}$. Thus, whenever $S_{i} \in \mathcal{S}^{\prime}$, then $p_{i}>p_{i-1}+1$ and we have $\operatorname{Dist}\left(G_{1}, G_{2}^{E}\left[p_{i-1}\right], G_{2}^{E}\left[p_{i-1}+1\right]\right) \geq M$ and

$$
\operatorname{Dist}\left(G_{1}, G_{2}^{E}\left[p_{i}-1\right], G_{2}^{E}\left[p_{i}\right]\right) \geq M
$$

since $G_{2}^{E}\left[p_{i-1}+1\right] \leq n$ and $G_{2}^{E}\left[p_{i}-1\right] \leq n$. It follows that $\left|\mathcal{S}^{\prime}\right| \leq \frac{2 s M}{2 M}=s$.

Theorem 6. There exists a constant $c>0$ such that ESAD (respectively, MSAD) admits no $\left(c \log \left|G_{1}\right|\right)$-approximation algorithm unless $\mathbf{P}=\mathbf{N P}$, where $\left|G_{1}\right|$ is the length of the smallest genome.

Proof. It is well known that the SETCOVER cannot be approximated within $(1-\varepsilon) \log n$ (where $n$ is the number of elements) for any $\varepsilon>0$ (see [26]) nor within $c \log m$ (where $m$ is the number of sets (see [27]) for some $c>0$ ). To be more precise, it has been proved in [27] that the instance of the Set Cover produced through the reduction in [26] is characterized by having $m \leq n^{5}$. Thus, for no $\varepsilon>0$, SETCOVER can be $(1-\varepsilon)$-approximated, even when restricting attention to instances in which $\log m \leq 5 \log n$. This means that there exists a constant $c^{\prime}$ such that no polynomial algorithm approximates SETCOVER within $c^{\prime}(\log m+\log n)$, with $c^{\prime}$ chosen small enough (consider any $c^{\prime}<\frac{1}{6}$ ). We claim that ESAD (respectively, MSAD) admits no $\left(\frac{c^{\prime}}{4} \log \left|G_{1}\right|\right)$-approximation algorithm. We proceed as follows: We assume that we are given a $\left(\frac{c^{\prime}}{4} \log \left|G_{1}\right|\right)$-approximation algorithm $A$ for ESAD (respectively, MSAD) and design a $c^{\prime}(\log m+$ $\log n$ )-approximation algorithm for SETCOVER which rests on algorithm $A$ as a subroutine. The theorem then follows from the above collected inapproximability facts about SETCOVER. After receiving in input an instance $\langle V, \mathcal{S}\rangle$ of SETCOVER, we construct the instance $\left\langle G_{1}, G_{2}\right\rangle$ of ESAD (respectively, MSAD) as described above. Notice that $\left|G_{1}\right| \leq 2 M$ and, hence,

$$
\log \left|G_{1}\right| \leq \log 2 m^{2} n^{2} \leq 3(\log m+\log n) .
$$

Let opt be the minimum size of a set cover for $\langle V, \mathcal{S}\rangle$. Then, by Lemma 9, there exists an exemplar subgenome $G_{2}^{E}$ of $G_{2}$ such that $\operatorname{SAD}\left(G_{1}, G_{2}^{E}\right) \leq 2$ opt $M+5 M$. By running algorithm $A$, we are hence guaranteed to find an exemplar subgenome $G_{a p x}^{E}$ of $G_{2}$ such that

$$
\begin{aligned}
\operatorname{SAD}\left(G_{1}, G_{a p x}^{E}\right. & \leq(2 \text { opt } M+5 M) \frac{c^{\prime}}{4} \log \left|G_{1}\right| \\
& \leq\left(\frac{8}{3} \text { opt } M\right) \frac{c^{\prime}}{4} 3(\log m+\log n) \\
& \leq(2 \text { opt } M) c^{\prime}(\log m+\log n) .
\end{aligned}
$$

Indeed, in the derivation of the above chain of inequalities, we can conveniently assume that the value of opt is sufficiently big since, if opt was bounded by any constant, then an optimal solution to the original SETCOVER instance could be found in polynomial time. Now, Lemma 10 (whose proof can be easily converted 
TABLE 1

Results Concerning the Exemplar Model

\begin{tabular}{|c|c|c|}
\hline \multicolumn{3}{|c|}{ Exemplar Model } \\
\hline Measure & "Complexity & " Approximability \\
\hline Breakpoints & $\begin{array}{c}\text { NP-complete [9] } \\
\text { even when } \operatorname{occ}\left(G_{1}\right)=1 \\
\text { and } \operatorname{occ}\left(G_{2}\right)=2\end{array}$ & $\begin{array}{c}\text { APX-hard [21] } \\
\text { even when } \operatorname{occ}\left(G_{1}\right)=1 \\
\text { and } \operatorname{coc}\left(G_{2}\right)=2\end{array}$ \\
\hline Reversals & $\begin{array}{c}\text { NP-complete [9] } \\
\text { even when } \operatorname{occ}\left(G_{1}\right)=2 \\
\text { and } \operatorname{coc}\left(G_{2}\right)=2\end{array}$ & $\begin{array}{c}\text { APX-hard [13] } \\
\text { even when } \operatorname{occ}\left(G_{1}\right)=2 \\
\text { and } \operatorname{coc}\left(G_{2}\right)=2\end{array}$ \\
\hline Conserved Intervals & $\begin{array}{c}\text { NP-complete [18] } \\
\text { even when } \operatorname{occ}\left(G_{1}\right)=1(*)\end{array}$ & $\begin{array}{c}\text { APX-hard [21] } \\
\text { even when } \operatorname{occ}\left(G_{1}\right)=1 \\
\text { and } \operatorname{coc}\left(G_{2}\right)=2\end{array}$ \\
\hline Common Intervals & $\begin{array}{l}\text { NP-complete (Theorem 1) } \\
\text { even when } \operatorname{occ}\left(G_{1}\right)=1 \\
\text { and } \operatorname{occ}\left(G_{2}\right)=2\end{array}$ & $\begin{array}{c}\text { APX-hard [21] } \\
\text { even when } \operatorname{occ}\left(G_{1}\right)=1 \\
\text { and } \operatorname{occ}\left(G_{2}\right)=2\end{array}$ \\
\hline MAD & $\begin{array}{l}\text { NP-complete (Theorem 5) } \\
\text { even when } \operatorname{occ}\left(G_{1}\right)=1 \\
\text { and } \operatorname{occ}\left(G_{2}\right) \leq 9\end{array}$ & $\begin{array}{l}\text { APX-hard (Theorem 5) } \\
\text { even when } \operatorname{occ}\left(G_{1}\right)=1 \\
\text { and } \operatorname{coc}\left(G_{2}\right) \leq 9\end{array}$ \\
\hline SAD & $\begin{array}{l}\text { NP-complete (Theorem 6) } \\
\text { even when } \operatorname{occ}\left(G_{1}\right)=1\end{array}$ & $\begin{array}{l}\text { APX-hard (Theorem 6) } \\
\text { even when } \operatorname{coc}\left(G_{1}\right)=1\end{array}$ \\
\hline
\end{tabular}

$\left({ }^{*}\right)$ We note that this result can actually be extended to the case where $\operatorname{occ}\left(G_{1}\right)=1$ and occ $\left(G_{2}\right)=2$ by reducing the problem from VERTEXCOVER instead of SETCOVER.

into a polynomial time algorithm) shows how, starting from $G_{a p x}^{E}$, one can obtain a set cover $S^{\prime}$ with $\left|\mathcal{S}^{\prime}\right| \leq$ $\frac{1}{2 M}(2$ opt $M) c^{\prime}(\log m+\log n)=$ opt $c^{\prime}(\log m+\log n)$.

\section{Summary of the Results and Discussion}

In this section, we give a summary of the results from this paper, as well as some other results concerning the complexity of computing several classical (dis)similarity measures, under both the exemplar and the matching models. We found it interesting to end this paper by giving an overview of the existing results in this area since several recent papers, by different groups of authors, have investigated the problem. Hence, in addition to the number of common intervals, MAD number and SAD number, we include results concerning the number of conserved intervals (initially defined in [23]), number of breakpoints, and number of reversals. However, we should note that the three above-mentioned measures take signs into account, which is not the case for common intervals, MAD, and SAD.

We recall that $\operatorname{occ}(G)$ denotes the maximum of $\operatorname{occ}(G, g)$ over all genes $g$ in $G$, where $\operatorname{occ}(G, g)$ denotes the maximum number of occurrences of a gene $g$ in genome $G$ (regardless of the signs). We also recall that $f(G)$ denotes the number of different families of genes that contain several occurrences in genome $G$.

The results concerning the exemplar model are summarized in Table 1, whereas the ones concerning the matching model are summarized in Table 2.

The main conclusion that we can draw from these two tables is that, as soon as $\operatorname{occ}\left(G_{1}\right)=1$ and $\operatorname{occ}\left(G_{2}\right)=2$, the computation of five out of the six above-mentioned measures becomes NP-complete under both the exemplar and matching models. In that sense, we are able to draw the exact border between polynomial problems $\operatorname{occ}\left(G_{1}\right)=\operatorname{occ}\left(G_{2}=1\right)$ and NP-complete problems $\left(\operatorname{occ}\left(G_{1}\right)=1\right.$ and $\left.\operatorname{occ}\left(G_{2}\right)=2\right)$, except for the number of reversals where a gap exists (we do
TABLE 2

Results Concerning the Matching Model

\begin{tabular}{|c|c|c|}
\hline \multicolumn{3}{|c|}{ Matching Model } \\
\hline Measure & Complexity & Approximability \\
\hline Breakpoints & $\begin{array}{c}\text { NP-complete } \\
\text { even when } \operatorname{occ}\left(G_{1}\right)=1 \\
\text { and } \operatorname{occ}\left(G_{2}\right)=2[9] \\
\text { even when } f\left(G_{1}\right)=f\left(G_{2}\right)=1[2]\end{array}$ & $\begin{array}{l}\text { APX-hard [21] } \\
\text { even when } \operatorname{occ}\left(G_{1}\right)=1 \\
\text { and } \operatorname{occ}\left(G_{2}\right)=2\end{array}$ \\
\hline Reversals & $\begin{array}{c}\text { NP-complete [10] } \\
\text { even when } \operatorname{occ}\left(G_{1}\right)=2 \\
\text { and } \operatorname{occ}\left(G_{2}\right)=2\end{array}$ & \\
\hline $\begin{array}{l}\text { Conserved } \\
\text { Intervals }\end{array}$ & $\begin{array}{c}\text { NP-complete } \\
\text { even when } o c c\left(G_{1}\right)=1[18](*) \\
\text { even when } f\left(G_{1}\right)=f\left(G_{2}\right)=1[18]\end{array}$ & $\begin{array}{c}\text { APX-hard [21] } \\
\text { even when } \operatorname{occ}\left(G_{1}\right)=1 \\
\text { and } \operatorname{occ}\left(G_{2}\right)=2\end{array}$ \\
\hline $\begin{array}{l}\text { Common } \\
\text { Intervals }\end{array}$ & $\begin{array}{c}\text { NP-complete } \\
\text { even when } \operatorname{occ}\left(G_{1}\right)=1 \\
\text { and } \operatorname{occ}\left(G_{2}\right)=2(\text { Theorem 1) } \\
\text { even when } f\left(G_{1}\right)=f\left(G_{2}\right)=1 \text { (Th. 2) }\end{array}$ & $\begin{array}{l}\text { APX-hard [21] } \\
\text { even when } \operatorname{occ}\left(G_{1}\right)=1 \\
\text { and } \operatorname{occ}\left(G_{2}\right)=2\end{array}$ \\
\hline MAD & $\begin{array}{c}\text { NP-complete (Theorem 5) } \\
\text { even when } \operatorname{occ}\left(G_{1}\right)=1 \\
\text { and } \operatorname{occ}\left(G_{2}\right) \leq 9\end{array}$ & $\begin{array}{l}\text { APX-hard (Theorem 5) } \\
\text { even when } \operatorname{occ}\left(G_{1}\right)=1 \\
\text { and } \operatorname{occ}\left(G_{2}\right) \leq 9\end{array}$ \\
\hline SAD & $\begin{array}{l}\text { NP-complete (Theorem 6) } \\
\text { even when } \operatorname{occ}\left(G_{1}\right)=1\end{array}$ & $\begin{array}{l}\text { APX-hard (Theorem 6) } \\
\text { even when } \operatorname{coc}\left(G_{1}\right)=1\end{array}$ \\
\hline
\end{tabular}

$\left({ }^{*}\right)$ We note that this result can actually be extended to the case where occ $\left(G_{1}\right)=1$ and occ $\left(G_{2}\right)=2$ by reducing the problem from VERTEXCOVER instead of SETCOVER.

not know the complexity of the problem when $\operatorname{occ}\left(G_{1}\right)=1$ and $\operatorname{occ}\left(G_{2}\right)=2$ ).

Another interesting parameter to consider for the complexity of those problems is $f(G)$, the number of families of genes that are duplicated in genome $G$. Concerning this parameter, only a few results are known (breakpoints and conserved and common intervals, in the matching model only).

Concerning the approximability of the problems, it turns out that, even when $\operatorname{occ}\left(G_{1}\right)=1$, we are able to say that five out of the six measures lead to APX-hard problems. For the number of reversals, it is APX-hard in the exemplar model when $\operatorname{occ}\left(G_{1}\right)=\operatorname{occ}\left(G_{2}\right)=2$ [13]. However, for three of those five cases (breakpoints and conserved and common intervals) similar to the complexity results, we know that the problem is APX-hard even when $\operatorname{occ}\left(G_{1}\right)=1$ and $\operatorname{occ}\left(G_{2}\right)=2$, whereas, in the others, the value of $\operatorname{occ}\left(G_{2}\right)$ is either unbounded (SAD) or bounded by constant 9 (MAD).

\section{CONCLUSION}

In this paper, we have investigated the algorithmic complexity of the problem of computing similarity measures between genomes in the case where they contain duplicates. This has been done for three measures: common intervals, MAD, and SAD. We have shown that the three problems are NP-complete, for both the exemplar and matching variants. Moreover, we have provided APX-hardness results concerning MAD and SAD. Those results, together with the ones concerning conserved intervals, breakpoints, and reversals, basically show that, as soon as duplicates are present, the problem becomes hard and even hard to approximate, even in very restricted instances.

Several lines of research would be interesting to follow, some of which we mention below:

- Make Tables 1 and 2 even more precise. In particular, 1) complete the cases for which no result 
is known or a gap exists (that is, the number of reversals), 2) study more deeply the complexity and approximability results with respect to the parameter $f$, and 3) tighten, if possible, the results concerning the (in)approximability of the problems, notably for the number of reversals in the exemplar model.

- Find Fixed-Parameter Tractable algorithms for those problems in order to circumvent NP-completeness and APX-hardness of the problems.

- Find good heuristics for those problems, as done, for instance, in [17] and [28] (among many others), in which the authors are able to compare their proposed heuristic to the exact results.

\section{REFERENCES}

[1] D. Sankoff, "Genome Rearrangement with Gene Families," Bioinformatics, vol. 15, no. 11, pp. 909-917, 1999.

[2] G. Blin, C. Chauve, and G. Fertin, "The Breakpoint Distance for Signed Sequences," Proc. Algorithms and Computational Methods for Biochemical and Evolutionary Networks (CompBioNets '04), pp. 3-16, 2004.

[3] D. Sankoff, G. Leduc, N. Antoine, B. Paquin, B. Lang, and R. Cedergren, "Gene Order Comparisons for Phylogenetic Inference: Evolution of the Mitochondrial Genome," Proc. Nat'l Academy of Sciences USA, vol. 89, no. 14, pp. 6575-6579, 1992.

[4] G. Bourque, E. Zdobnov, P. Bork, P. Pevzner, and G. Tesler, “Comparative Architectures of Mammalian and Chicken Genomes Reveal Highly Variable Rates of Genomic Rearrangements across Different Lineages," Genome Research, vol. 15, no. 1, pp. 98110, 2005.

[5] D. Sankoff, "Gene and Genome Duplication," Current Opinion in Genetics Development, vol. 11, no. 6, pp. 681-684, 2001.

[6] J. Korbel, D. Snel, M. Huynen, and P. Bork, "Shot: A Web Server for the Construction of Genome Phylogenies," Trends in Genetics, vol. 18, no. 3, pp. 158-162, 2002.

[7] E. Belda, A. Moya, and F. Silva, "Genome Rearrangement Distances and Gene Order Phylogeny in $\gamma$-Proteobacteria," Molecular Biology Evolution, vol. 22, no. 6, pp. 1456-1467, 2005.

[8] G. Blin, A. Chateau, C. Chauve, and Y. Gingras, "Inferring Positional Homologs with Common Intervals of Sequences," Proc. RECOMB Int'l Workshop Comparative Genomics (RCG '06), pp. 2438, 2006.

[9] D. Bryant, "The Complexity of Calculating Exemplar Distances," Comparative Genomics: Empirical and Analytical Approaches to Gene Order Dynamics, Map Alignment, and the Evolution of Gene Families, pp. 207-212, Kluwer Academic, 2000.

[10] X. Chen, J. Zheng, Z. Fu, P. Nan, Y. Zhong, S. Lonardi, and T. Jiang, "Assignment of Orthologous Genes via Genome Rearrangement," IEEE/ACM Trans. Computational Biology and Bioinformatics, vol. 2, no. 4, pp. 302-315, Oct.-Dec. 2005.

[11] K. Swenson, M. Marron, J. Earnest-DeYoung, and B. Moret, "Approximating the True Evolutionary Distance between Two Genomes," Proc. Seventh Workshop Algorithm Eng. and Experiments and Second Workshop Analytic Algorithmics and Combinatorics (ALENEX/ANALCO '05), pp. 121-129, 2005.

[12] N.C. Thach, "Algorithms for Calculating Exemplar Distances," honours Year Project Report, Nat'1 Univ. of Singapore, 2005.

[13] Z. Chen, B. Fu, and B. Zhu, "The Approximability of the Exemplar Breakpoint Distance Problem," Proc. Second Int'l Conf. Algorithmic Aspects in Information and Management (AAIM '06), pp. 291-302, June 2006.

[14] M. Marron, K. Swenson, and B. Moret, "Genomic Distances under Deletions and Insertions," Theoretical Computer Science, vol. 325, no. 3, pp. 347-360, 2004.

[15] J. Tang and B. Moret, "Phylogenetic Reconstruction from GeneRearrangement Data with Unequal Gene Content," Proc. Eighth Int'l Workshop Algorithms and Data Structures (WADS '03), pp. 3746, 2003.
[16] Z. Fu, X. Chen, V. Vacic, P. Nan, Y. Zhong, and J. Tang, "A Parsimony Approach to Genome-Wide Ortholog Assignment," Proc. 10th Ann. Int'l Conf. Research in Computational Molecular Biology (RECOMB '06), pp. 578-594, Apr. 2006.

[17] C. Nguyen, Y. Tay, and L. Zhang, "Divide-and-Conquer Approach for the Exemplar Breakpoint Distance," Bioinformatics, vol. 21, no. 10, pp. 2171-2176, 2005.

[18] G. Blin and R. Rizzi, "Conserved Interval Distance Computation between Non-Trivial Genomes," Proc. 11th Ann. Int'l Conf. Computing and Combinatorics (COCOON '05), pp. 22-31, Aug. 2005.

[19] G. Bourque, Y. Yacef, and N. El-Mabrouk, "Maximizing Synteny Blocks to Identify Ancestral Homologs," Proc. RECOMB Int'l Workshop Comparative Genomics (RCG '05), pp. 21-34, Sept. 2005.

[20] Z. Chen, R. Fowler, B. Fu, and B. Zhu, "Lower Bounds on the Approximation of the Exemplar Conserved Interval Distance Problem of Genomes," Proc. 12th Ann. Int'l Conf. Computing and Combinatorics (COCOON '06), pp. 245-254, 2006.

[21] S. Angibaud, G. Fertin, and I. Rusu, On the Inapproximability of Similarity Measures between Genomes Containing Duplicates, 2006,

[22] D. Sankoff and L. Haque, "Power Boosts for Cluster Tests," Proc. RECOMB Int'l Workshop Comparative Genomics (RCG '05), pp. 121130, Sept. 2005.

[23] A. Bergeron and J. Stoye, "On the Similarity of Sets of Permutations and Its Applications to Genome Comparison," Proc. Ninth Ann. Int'l Conf. Computing and Combinatorics (COCOON '03), pp. 68-79, July 2003.

[24] M. Garey and D. Johnson, Computers and Intractability: A Guide to the Theory of NP-Completeness. W.H. Freeman, 1979.

[25] C.H. Papadimitriou and M. Yannakakis, "Optimization, Approximation, and Complexity Classes," J. Computer and System Sciences, vol. 43 , pp. $425-440,1991$.

[26] U. Feige, "A Threshold of $\ln (n)$ for Approximating Set Cover," J. ACM, vol. 4, no. 45, pp. 634-652, 1998.

[27] S. Eidenbenz, C. Stamm, and P. Widmayer, "Positioning Guards at Fixed Height above a Terrain-An Optimum Inapproximability Result," Proc. Sixth European Symp. Algorithms (ESA'98), pp. 187198, 1998.

[28] S. Angibaud, G. Fertin, I. Rusu, and S. Vialette, "How PseudoBoolean Programming Can Help Genome Rearrangement Distance Computation," Proc. RECOMB 2006 Int'l Workshop Comparative Genomics (RCG '06), pp. 75-86, 2006.

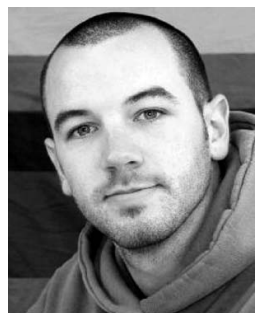

Guillaume Blin received the PhD degree in computer science from Nantes University, France, in 2005. He is an associate professor in the Gaspard Monge Institute, University of Marne-La-Vallée, France. His current research interests include computational complexity and approximation, algorithms, and bioinformatics.

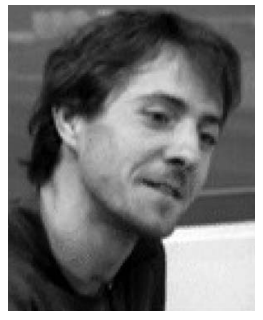

Cedric Chauve received the $\mathrm{PhD}$ degree in computer science from the University of Bordeaux I, France, in 2000. From 2001 to 2006, he was a professor in the Department of Computer Science at the University of Québec in Montréal. $\mathrm{He}$ is now an associate professor in the Department of Mathematics at Simon Fraser University, Burnabay, British Columbia, Canada. His current research interest is the development of combinatorial models and efficient algorithms for comparative genomics.

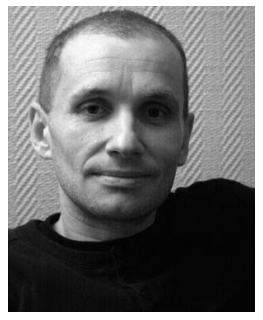

Guillaume Fertin received the $\mathrm{PhD}$ degree in computer science from the University of Bordeaux, France, in 1999. He is a professor in the Laboratoire d'Informatique de Nantes-Atlantique (LINA) at the University of Nantes, France. His current research interests are optimization, discrete mathematics, computational complexity, and algorithms dedicated to bioinformatics. 


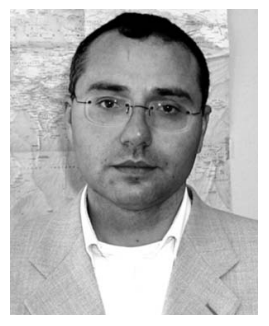

Romeo Rizzi received the Laurea degree in electronic engineering from the Politecnico di Milano in 1991 and the $\mathrm{PhD}$ degree in computational mathematics and informatics from the University of Padova, Italy, in 1997. Afterward, he held postdoctoral and other temporary positions at research centers like Centrum voor Werk en Inkomen (CWI; Amsterdam), Basic Research in Computer Sciences (BRICS; Aarhus, Denmark), and Istituto per la Ricerca Scientifica e Tecnologica (IRST, Trento, Italy). In 2001, he became an assistant professor at the University of Trento. Since 2005, he has been an associate professor at the University of Udine. He has a background in operations research and his main interests are in combinatorial optimization and algorithms. He is an area editor of $4 O R$ and acts as a reviewer for the American Mathematical Society. He has published more than 40 articles in a broad range of scientific journals in the areas of discrete mathematics, combinatorics, and algorithms. Since 2004, he has been a trainer of the Italian team for the iOi.

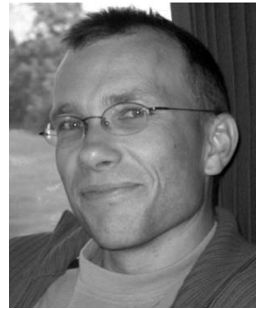

Stéphane Vialette received the PhD degree in computer science from the University Paris 7 in 2001. He has been a postdoctoral researcher at the Laboratoire de Génétique Moléculaire at the Ecole Normale Supérieure for two years. Since 2003, he has been an assistant professor with the Bioinformatics Group from the Computer Science Research Laboratory (LRI) at the Université Paris-Sud 11. His research rithmics, and combinatorics. interests include computational biology, algo-

$\triangleright$ For more information on this or any other computing topic, please visit our Digital Library at www.computer.org/publications/dlib. 